\title{
eJRIEPS
}

Ejournal de la recherche sur l'intervention en éducation physique et sport

$18 \mid 2009$

Varia

\section{Connaissances et expertise perceptive des entraîneurs en gymnastique artistique}

\section{Cathy Rolland et Marc Cizeron}

\section{OpenEdition}

\section{Journals}

Édition électronique

URL : http://journals.openedition.org/ejrieps/5507

DOI : 10.4000/ejrieps.5507

ISSN : 2105-0821

Éditeur

ELLIADD

\section{Référence électronique}

Cathy Rolland et Marc Cizeron, « Connaissances et expertise perceptive des entraîneurs en gymnastique artistique », eJRIEPS [En ligne], 18 | 2009, mis en ligne le 01 juillet 2020, consulté le 18 mars 2021. URL : http://journals.openedition.org/ejrieps/5507 ; DOI : https://doi.org/10.4000/ejrieps. 5507

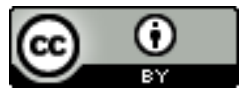

La revue eJRIEPS est mise à disposition selon les termes de la Creative Commons Attribution 4.0 International License. 
Connaissances et expertise perceptive des entraîneurs en gymnastique artistique

Cathy Rolland \& Marc Cizeron

UFR STAPS Clermont-Ferrand II, Laboratoire PAEDI Université Blaise Pascal ClermontFerrand II

\section{Résumé}

L'étude présentée porte sur l'activité des entraîneurs experts en gymnastique artistique et cherche à décrire et comprendre les ressources cognitives grâce auxquelles ils interviennent auprès des gymnastes pour transformer leurs habiletés gymniques. S'agissant de l'étude de connaissances en tant qu'elles sont investies dans les situations pratiques, le cadre théorique retenue est celui de l'anthropologie cognitive située. Les matériaux ont été élaborés à partir d'une démarche qualitative fondée sur une étude de cas. Les résultats révèlent que l'activité des entraîneurs est une activité d'interprétation permanente des réalisations des gymnastes à l'aide de repères, de structures d'intelligibilité du mouvement et non une activité de reconnaissance de formes relativement à un modèle formel. Ces structures d'intelligibilité pourraient constituer des archétypes énactés du savoir-faire gymnique.

«Doué du coup d'œil du maquignon, il était le sorcier du mouvement. » Claude Piard reconnaît à travers l'usage d'une expression consacrée dans le domaine de l'entraînement sportif, la capacité de l'entraîneur expert en gymnastique artistique, de savoir " toujours "voir exact" dans la production gestuelle d'un élève et fournir toujours la solution adéquate au problème posé. » (Piard, 1992, p.139)

L'intervention des entraîneurs en gymnastique artistique, finalisée par le modelage précis du corps en mouvement sur les agrès (Rolland \& Cizeron, 2008), peut être décrite comme une alternance de moments d'observation du mouvement et de moments d'interactions avec les gymnastes. Parce que l'entraîneur expert évolue dans un monde de mouvements gymniques qu'il s'est rendu familier (Cizeron, 2002), il perçoit dans ceux-ci des éléments qui lui permettent d'intervenir pour les transformer. Si l'expérience pratique des entraîneurs est communément reconnue comme le fondement de leurs compétences professionnelles, il semble alors pertinent de s'intéresser à ces connaissances implicites, non formalisées, qui s'expriment dans l'action. Cet intérêt conduit à rompre avec le 
" modèle de la rationalité technique » (Schön, 1983) qui envisage l'intervention comme la résolution de problèmes pratiques grâce à l'application formelle de connaissances dans l'action. Ce modèle peine en effet à expliquer la façon dont les acteurs agissent dans des contextes naturels nécessairement changeants, imprévisibles et problématiques. D'autre part, en centrant l'attention sur des connaissances formalisées a priori, c'est-à-dire en dehors de leur inscription dans l'expérience vécue, il tend à perdre le caractère expérientiel de ces connaissances.

\section{Les connaissances des entraîneurs}

Plusieurs auteurs (Malglaive, 1990 ; Le Boterf, 1998) distinguent différentes catégories de connaissances contribuant à la compétence. Une différenciation globale et consensuelle entre les auteurs s'établit entre connaissances théoriques et connaissances pratiques. Dans le domaine de l'entraînement sportif, l'activité des entraîneurs est généralement rationalisée et légitimée en la référant à des connaissances scientifiques. Dans le domaine de l'entraînement en gymnastique sportive, la démarche adressée aux entraîneurs postule que la compréhension des habiletés gymniques suppose des connaissances biomécaniques du mouvement. C'est précisément le cas dans le Mémento des Activités Gymniques (1997) où sont présentées des analyses descriptives, biomécaniques et musculaires précises des principales habiletés à chaque agrès. C'est aussi typiquement la démarche de Pernet (1994) pour qui les entraîneurs doivent posséder une connaissance parfaite des techniques gymniques. L'idée de connaissance parfaite est rapportée à celle d'une forme canonique des habiletés et leur compréhension biomécanique. La culture de l'entraînement en gymnastique sportive s'en trouve fortement pénétrée de référence à la biomécanique. Dans l'ouvrage de Smith consacré à cet aspect, il est précisé que «l'étude du mouvement gymnique à partir des principes mécaniques déterminera les positions correctes du corps et les mouvements nécessaires à leur bonne exécution 》 (Smith, 1991, p. 20). La correction et la bonne exécution apparaissent comme des normes faisant office de référence pour les entraîneurs, normes à l'appui desquelles ils auraient à transformer progressivement les mouvements des gymnastes. Néanmoins une étude portant sur les connaissances des enseignants et entraîneurs experts en gymnastique a montré que les références qu'ils faisaient à la biomécanique pour justifier leurs interventions auprès des gymnastes étaient essentiellement approximatives et métaphoriques (Cizeron, 2002). En effet, lorsqu'ils parlent de biomécanique, ils utilisent des métaphores comme celle par exemple de l'énergie comme " substance fluide " susceptible de circuler dans le corps. Ils mobilisent des images comme celle du "bâton articulé », ou du «crayon » comme 
substituts aux concepts de la mécanique (celui de «force » par exemple) pour parler du gainage ou de la rigidité du corps.

L'étude présentée porte sur l'activité des entraîneurs experts en gymnastique artistique et cherche à décrire et comprendre les ressources cognitives grâce auxquelles ils interviennent auprès des gymnastes pour transformer leurs habiletés gymniques.

\section{Cadre théorique et méthodologique}

Le cadre théorique retenu se réclame d'une anthropologie cognitive située (Theureau, 1992). L'anthropologie cognitive porte l'accent sur la description et l'analyse des connaissances à la fois implicites et tacites qui structurent les activités humaines. Son inscription dans le paradigme de l'action et de la cognition « situées " (Suchman, 1987 ; Greeno, 1998 ; Kirshner et Whistson, 1997) se rapporte à quatre idées majeures qui marquent une rupture fondamentale avec les présupposés cognitivistes classiques (Saury, Ria, Sève, \& Gal-Petitfaux, 2006) .

- La cognition a un caractère incarné irréductible. Elle s'ancre dans le corps où s'entremêlent de façon syncrétique des dimensions perceptives, émotionnelles, sensori-motrices.

- L'action et la situation sont structurellement couplées dans le sens où elles sont réciproquement déterminées. Le caractère asymétrique de ce couplage fait que l'acteur construit à chaque instant sa propre situation. Celle-ci est ainsi le résultat d'une interprétation continue de ce que vit l'acteur à chaque instant.

- L'action a un caractère essentiellement indéterminé et opportuniste. Tout particulièrement dans les environnements naturels et complexes, les acteurs interprètent en permanence le sens des situations qu'ils vivent et exploitent les ressources que leur offre l'environnement pour agir. Le sens de ce qu'ils font et les ressources qu'ils exploitent pour agir émergent en partie en cours d'action.

- La cognition est conçue comme socialement et culturellement située. Les significations qui configurent l'action sont ancrées dans un contexte culturel et social. De ce point de vue, l'action n'est jamais en situation d'apesanteur culturelle, elle véhicule des significations partagées, déposées dans des discours, des conventions, des objets, etc.

La méthodologie retenue relève du cadre général des études de cas (Passeron \& Revel, 2005). Elle visait à décrire et analyser l'activité de l'entraîneur en l'observant dans des situations écologiques les plus ordinaires possible. Le terrain de l'étude est celui de l'entraînement sportif de haut niveau : un club sportif de niveau national, ainsi que deux 
centres nationaux de la Fédération Française de Gymnastique (FFG). Deux entraîneurs féminins et dix entraîneurs masculins ont été suivis au cours de leur activité professionnelle auprès de gymnastes de haut niveau âgés de 7 à 23 ans. Les entraîneurs sont identifiés dans la suite du texte par leurs initiales de $A$ à $L$.

Afin de croiser des données en première et en troisième personne (Vermersch, 1994), deux types de matériaux complémentaires ont été élaborés :

i) des données d'observation du flux de comportements, c'est-à-dire les comportements observables de l'entraîneur au cours de l'entraînement tels que ses communications verbales, ses placements et déplacements, ses gestes, ses manipulations corporelles des gymnastes. Ces données recueillies à l'aide de support papier, audio et vidéo constituaient des traces de l'activité. Présentées à l'entraîneur, elles lui permettaient de se focaliser sur son activité réelle passée afin de l'expliciter.

ii) des verbalisations réflexives produites par l'entraîneur au cours d'entretiens menés dans un délai très court après une séquence d'entraînement. Les questions invitaient l'entraîneur à décrire ce qu'il repérait comme particulièrement significatif, à discerner ce qu'il percevait directement ou par inférence, à préciser ce qu'il percevait et les liens établis avec les prescriptions de transformations qu'il proposait au gymnaste.

Une méthodologie originale a été développée, consistant dans le rapprochement entre la technique de l'instruction au sosie (Oddone et al., 1981) et l'observation participante ; elle peut ainsi être qualifiée d' " instruction au pair ». Celle-ci se caractérise par une situation d'entretien au cours de laquelle l'entraîneur adresse son discours à un pair reconnu (en l'occurrence, le chercheur, qui est aussi un entraîneur-gymnaste reconnu comme tel par l'entraîneur interviewé), pour l'instruire de son savoir. Elle permet l'élaboration de données en deuxième personne (Vermersch, 1994) qui relèvent de ce que peut comprendre, ressentir avec empathie le chercheur de l'expérience subjective de l'entraîneur.

Les matériaux recueillis ont fait l'objet d'une analyse qualitative inductive. La méthode comparative continue (Strauss, 1992) a été utilisée afin de produire des résultats théoriques intégrés aux matériaux recueillis.

\section{Résultats}

Les entraîneurs experts expliquent observer le mouvement gymnique à l'aide d'un type idéal de formes corporelles. Ils évoquent une activité de comparaison à un modèle par l'utilisation d'un "calque » abstrait du mouvement qu'ils juxtaposent mentalement au mouvement concret du gymnaste qu'ils observent. 
" J'ai un calque dans la tête et je compare ce qu'elle fait par rapport à ce calque, s'il y a un décalage » Entraîneur $B$.

L'analyse conjuguée des prescriptions et explications que les entraîneurs ont adressées aux gymnastes et les explicitations fournies au cours des entretiens ont permis de questionner cette catégorie indigène. Elle révèle que l'activité des entraîneurs est une activité d'interprétation permanente des réalisations des gymnastes à l'aide de repères, de structures d'intelligibilité du mouvement et non une activité de reconnaissance de formes relativement à un modèle formel. Ces structures d'intelligibilité pourraient constituer des archétypes des différentes habiletés gymniques. La notion d'archétype est provisoirement choisie car elle permet de rendre compte tout à la fois des caractères typiques qui organisent la réalisation d'un gymnaste et de la dimension singulière, originale de cette réalisation. En effet, un archétype par définition, partage avec la totalité des objets particuliers affiliés à celui-ci l'intégralité de ses caractéristiques. La réciproque est fausse car l'objet particulier partage quelques unes de ses caractéristiques (les principales ou les plus représentatives de ce qu'il est) avec son archétype tout en conservant ses caractéristiques uniques qui en font un objet singulier.

\section{1. Caractéristiques des typifications perceptives}

Les résultats de l'étude montrent que les entraîneurs experts jugent les réalisations des gymnastes à l'aide de traits typiques organisateurs de chaque habileté gymnique. Ces traits typiques sont des formes idéales typiques du corps qui correspondent à ce qu'ils nomment des « phases de placement ». En effet, l'activité perceptive des entraîneurs présente la caractéristique de discrétiser le mouvement en séquences. L'entraîneur B expose le caractère séquentiel du calque qu'il utilise pour observer les habiletés:

"Sur la vrille arrière, c'est net je fais comme ça, je compare au calque. Je vois bien les différentes phases ».

Ces séquences sont des "phases de placement ", des formes transitoires comme composantes de formes plus complexes (Rolland, 2004).

3. 1. 1. Des formes corporelles comme totalités signifiantes Dans leur aspect observable, ces phases de placement sont des séquences de formes corporelles, la forme étant définie comme l'apparence extérieure résultant de dispositions des parties c'est-à-dire les segments et entités corporels du gymnaste. Les représentations de ces phases fournies aux gymnastes au cours de l'activité de transmission pourraient conforter cette acception puisqu'elles montrent le corps dans une position statique sur l'agrès. 
Suite à leur réalisation, les entraîneurs informent les gymnastes sur les phases de placement telles qu'elles devraient selon eux être réalisées et/ou telles qu'ils les ont perçues. Ils font usage pour cela de différents médias de représentation : leur propre corps, leur main et des supports plans tels que tableaux, tapis sur lesquels ils dessinent des représentations graphiques. Ces tracés représentent le corps en mouvement comme un assemblage de portions de droite, de courbes, qui constituent une forme globale continue ou brisée. La main présentée de profil au gymnaste dessine aussi une forme géométrique, l'articulation métacarpo-phalangienne étant utilisée pour séparer le haut du corps du bas du corps, le buste des jambes. II est remarquable de noter que ces représentations montrent quasi-systématiquement le gymnaste de profil. Cette orientation spatiale privilégiée rend visible des placements ou déplacements du corps dans le plan sagittal, plan dans lequel se déroule en grande partie les déplacements gymniques sur les agrès. Ces représentations sont significatives de ce qui est pertinent pour les entraîneurs dans le mouvement gymnique pour le transformer. Nous allons voir que ce qui est pertinent pour eux dépasse largement l'agencement topographique des différentes parties corporelles. En effet, ces représentations constituent des figurations de propriétés.

Les phases de placement présentent pour les entraîneurs différentes propriétés : géométriques, topologiques, dynamiques et des propriétés liées à l'organisation temporelle et rythmique. Les propriétés géométriques correspondent au positionnement dans l'espace des segments corporels les uns par rapport aux autres, ce qui dessine pour les entraîneurs des lignes, des angles, des courbes. L'entraîneur C rappelle à ses gymnastes les deux exigences pour réaliser l'habileté gymnique roue (représentation schématisée ci-dessous) :

"les bras comme ça (elle montre en plaçant ses bras à la verticale) et puis? Les fesses serrées oui (première figure de la représentation ci-dessous)».

Les explications fournies au chercheur à propos de ses exigences montrent que le corps apparaît comme un corps géométrisé :

"Si elles recherchent cet alignement au départ et à l'arrivée, ça veut dire qu'elles sont restées pendant toute la roue alignées. Moi ce que je veux, c'est qu'il n'y ait pas à un moment donné qu'elles ferment l'angle des bras, qu'elles cherchent l'alignement sans arrêt ». 


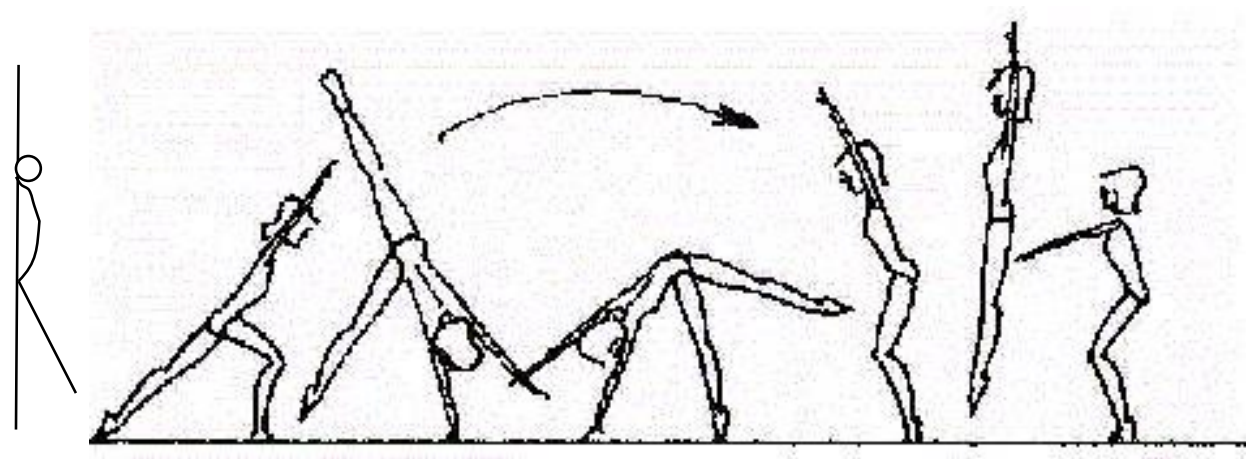

Figure 1: Représentation schématisée de l'habileté gymnique roue dans un code de référence avec ajout de la phase de placement enseignée par les entraîneurs : $1^{\text {ère }}$ figure.

Cette image d'un corps géométrisé intègre pour les entraîneurs des dimensions topologiques et dynamiques. Les propriétés topologiques indiquent le positionnement de la forme géométrique dans l'espace d'évolution, agrès ou matériel, ou par rapport aux axes vertical et horizontal que l'entraîneur imagine pour repère. Au cours de l'habileté représentée ci-dessus, l'entraîneur C demande aux gymnastes à la pose des mains au sol de "s'aligner contre un mur ».

Les propriétés dynamiques ont trait aux actions du gymnaste pour réaliser la phase de placement. L'entraîneur $\mathrm{C}$ précise au chercheur à propos de la première séquence :

"Ce n'est pas qu'une position. Je veux les voir vraiment grandies. On leur demande d'être vraiment actives au niveau du grandissement des épaules".

La phase de placement, en tant qu'élément discret constitutif du mouvement, présente pour les entraîneurs une structuration temporelle et rythmique particulière. Cette propriété décrit le moment de déclenchement de la phase de placement, son temps de maintien et organise la succession dans le temps des différentes séquences. L'entraîneur I explique l'organisation temporelle d'une action qui doit amener le gymnaste vers une phase de placement constitutive d'une habileté gymnique (balancé avant $1 / 2$ tour) aux barres parallèles :

"Cette action, plus tôt. Cette action, tu dois l'emmener quand tu es en balancé arrière...et tu la termines à la fin du balancé avant ». 
Les intonations de ses explications verbales conjointes à une représentation manuelle du mouvement précisent aux gymnastes les temps forts et faibles, courts et longs du mouvement :

"Là, là, là...et là je jette la barre " (prononcé très vite et avec une voix plus forte).

Les formes corporelles figurées qui structurent les habiletés gymniques sont donc signifiantes pour les entraîneurs. Nous allons voir que les propriétés des phases de placement s'organisent pour eux en systèmes cohérents.

3. 1. 2. Des totalités signifiantes dans une configuration singulière et dynamique du mouvement

Le mouvement gymnique s'organise typiquement pour les entraîneurs experts selon une cohérence fondée sur des relations logiques d'ordre causal. Les relations logiques qu'ils établissent présentent un aspect diachronique et synchronique.

Dans leur aspect diachronique, les phases de placement ordonnent pour les entraîneurs le mouvement selon une causalité historique, l'avant déterminant l'après. Les entraîneurs identifient une dépendance chronologique entre phases de placement, les propriétés d'une phase étant déterminées par celles de la phase qui la précède. Les explications adressées par l'entraîneur I à un gymnaste lors d'un exercice aux barres parallèles illustrent ce résultat :

"si tu fais un tour et que tu enlèves la base, ça fait quoi ? ça tombe! C'est quoi ta base dans cet élément? Le balancé avant. Après tu fais demi-tour, demi-tour, équilibre dur. Si tu ne fais pas le balancé jusqu'au bout, y'a pas la suite, c'est logique ".

La dépendance chronologique existe également pour les entraîneurs entre éléments gymniques enchaînés. Ainsi, l'entraîneur $\mathrm{J}$ ayant observé une vrille avant au sol réceptionnée non sur les pieds mais sur les fesses, intervient verbalement auprès du gymnaste en lui demandant de transformer la réalisation de l'élément qui précède :

" il faut régler ton amplitude dans le salto avant tendu pour pouvoir enchaîner, ni trop, ni trop peu. Si tu es trop haut, tu arrives les épaules penchées en avant et tu piques du nez, et ta vrille est trop basse ".

La connaissance de cet ordre causal leur permet de trouver en amont, dans une phase antécédente non directement perçue mais logiquement inférée, l'explication d'un effet perçu du mouvement gymnique. L'entraîneur $\mathrm{J}$ s'adresse verbalement au gymnaste suite à sa réalisation d'une habileté gymnique aux barres parallèles : 
"tu ne fais rien avec les bras, donc les épaules partent en arrière et ça lâche ». Invité à expliciter la façon dont il voyait l'action des bras, l'entraîneur précise : " je ne vois pas le trajet des bras. Je vois plutôt la conséquence et je sais que c'est dû à cela ”.

La dépendance chronologique entre phases de placement, identifiée par les entraîneurs, organise la façon dont ils interviennent auprès des gymnastes. Par exemple, ils interrompent parfois leur observation du mouvement dès qu'ils perçoivent un décalage entre la réalisation en cours et leurs attentes. Leurs interventions auprès des gymnastes s'effectuent relativement à cette organisation chronologique. Par exemple, l'entraîneur I intervient à propos d'une habileté aux barres parallèles :

"lorsque le balancé est fait, on peut regarder l'étape suivante ».

Les relations causales que les entraîneurs établissent s'expriment également à un niveau synchronique, c'est-à-dire au sein de chaque phase de placement. Chaque phase constitue pour eux un agencement cohérent de propriétés. L'étude révèle que les entraîneurs caractérisent les phases de placement en leur attribuant des propriétés cohérentes entre elles, à partir d'éléments discrets perçus comme la position d'une seule entité corporelle ou sa situation spatiale par rapport à des repères matériels ou par rapport à une autre partie du corps. L'entraîneur J voit par exemple dans une phase de placement une position particulière de la tête et associe ce positionnement à des propriétés particulières de la phase qui génère typiquement un mouvement caractéristique du corps. :

"Si tu baisses la tête, tu abaisses le buste et tu ne peux pas monter ».

Les entraîneurs de niveau national manifestent une compréhension du mouvement gymnique organisée par des relations causales qui impliquent des séquences particulières des habiletés gymniques. Ils s'appuient sur cet archétype empirique de causalité pratique pour transformer les habiletés gymniques : en agissant sur une propriété, ils agissent sur les autres; en agissant sur une phase de placement, ils agissent sur les phases qui suivent. Ils développent une intelligibilité du mouvement fondée sur une connaissance cinématique empirique du mouvement. Cette connaissance est pragmatique, finalisée par la transformation des habiletés gymniques.

\section{1. 3. Logique fonctionnelle des typifications en formes transitoires}

Les séquences sur lesquelles les entraîneurs se focalisent au cours de leurs interventions auprès des gymnastes sont typiques de la manière dont ils connaissent les habiletés gymniques. Elles révèlent que pour les entraîneurs, faire apprendre une habileté gymnique consiste à faire transiter le gymnaste de phases en phases. En effet, les phases de placement sont appréhendées comme formes transitoires. Elles constituent tout en 
premier lieu des étapes spatiales vers la phase suivante en «plaçant» le corps du gymnaste de manière optimale pour poursuivre sa trajectoire. Invité à préciser le lien qu'il effectue entre la hauteur du bassin demandée au gymnaste à la fin d'un balancé avant aux barres parallèles et la réussite de l'exercice, l'entraîneur I explique :

«le bassin doit se placer suffisamment haut pour enclencher le 1/2 tour. Le bassin, c'est le cheminement vers le $1 / 2$ tour ».

Les interventions des entraîneurs visent à favoriser le repérage par les gymnastes de ces positionnements spatiaux et topologiques sur les agrès. Elles consistent parfois à faire apprendre ces phases indépendamment d'une réalisation globale, parfois de manière statique, décontextualisée du mouvement. Les entraîneurs justifient ces procédés par une connaissance du fonctionnement des gymnastes qui ont tendance selon eux, à anticiper, à valoriser les actions de rotation du corps au détriment des placements adaptés. L'entraîneur B illustre cette idée au travers des remarques adressées à un gymnaste concernant une habileté au saut :

“Tu t'es précipité dans l'acrobatie. L'intérêt ici, c'est de remonter les épaules avant de carper, avant de faire ton salto. II y a une anticipation sur le salto ».

Ce repérage statique des phases de placement nuit parfois à la coordination avec les autres phases lors de la réalisation du mouvement global. Les prescriptions des entraîneurs visent alors à constituer les phases comme étapes, transitions dont l'organisation temporelle et rythmique est au service de la réalisation globale. La prescription donnée par l'entraîneur B concernant la même habileté que ci-dessus vise une transformation inverse à l'adresse précédente :

" ça ne se décompose plus ; ça c'est l'éducatif. Maintenant, en même temps, tu redresses et tu carpes ».

La discrétisation des habiletés en séquences signifiantes constitue une connaissance typique mobilisée par les entraîneurs pour transformer les habiletés. Elle assurerait selon les entraîneurs des réalisations évolutives et en conséquence prometteuses de performances élevées.

3. 2. Des archétypes énactés de gestalts dynamiques

3. 2. 1. Le caractère énacté des interprétations des entraîneurs

Les entraîneurs interviennent sur des phases de placement qui acquièrent une signification pour eux dans la dynamique globale du mouvement et dans la situation et les interactions vécues au cours de l'entraînement. Le caractère archétypal attribué aux réalisations des gymnastes par les entraîneurs permet de rendre compte de la dimension située de l'activité perceptive et transformative des entraîneurs. En effet, les entraîneurs 
ne réagissent pas de la même manière à deux comportements apparemment identiques des gymnastes lors de la réalisation d'une même habileté. Ils interprètent constamment ces comportements à partir des significations qu'ils leur attribuent in situ. Ces interprétations prennent en compte les caractéristiques morphologiques singulières de chaque gymnaste ainsi que ses ressources telles que force, souplesse, dynamisme. Ainsi, ce n'est par exemple pas un angle mesurable qui est perçu par l'entraîneur lorsqu'il observe un gymnaste mais un angle dans la configuration dynamique et singulière d'une réalisation. Par exemple, le placement des épaules par rapport à l'appui des mains lors d'une prise d'élan en barres parallèles fait l'objet d'une intervention transformative de l'entraîneur s'il est jugé non efficace pour la réalisation de l'habileté gymnique globale, relativement aux qualités physiques du gymnaste. L'entraîneur I précise que :

"le contrôle avec les épaules en avant dans la descente de l'équilibre est fonction des qualités physiques. M., il est capable musculairement de résister à la vitesse, à la pression tandis que D., s'il fait ça, ses épaules vont partir en arrière; alors, je lui demande de contrôler en avançant les épaules. ”

Cette réalisation est également perçue dans un contexte singulier tel que l'état de fatigue des gymnastes, la période de l'année et la proximité ou pas d'échéances compétitives. L'entraîneur $\mathrm{K}$ est amené à expliciter pourquoi il n'intervient pas sur une habileté gymnique alors que la réalisation du gymnaste n'est a priori pas conforme aux prescriptions habituellement formulées :

"je ne lui dis rien parce qu'il n'y a pas de fautes, mais techniquement il ferme les jambes trop tôt. C'est son troisième jour d'entraînement ; il a été arrêté dix jours parce qu'il a été malade ».

Cet extrait montre également que le rapport des entraîneurs aux normes qu'ils évoquent sous le terme technique n'est pas un rapport de soumission, comme ce peut être le cas pour les juges par rapport au code de notation, mais un rapport d'usage. Ce sont des normes pratiques.

Les entraîneurs font émerger au cours de l'activité d'entraînement un monde signifiant qui intègre le cours de l'histoire de leurs interactions continues avec les gymnastes. Les archétypes présentent ainsi la caractéristique d'être énactés au cours de l'activité d'intervention auprès des gymnastes. Cette activité créatrice des entraîneurs se révèle notamment à travers les placements qu'ils adoptent pour observer le mouvement gymnique et se rendre perceptible des aspects constitutifs du mouvement. Les entraîneurs agissent sur les gymnastes en les faisant se mouvoir selon certaines prescriptions et proscriptions et privilégient alors un placement particulier par rapport au gymnaste afin de 
percevoir les transformations demandées et ses conséquences sur le mouvement. Si les transformations demandées concernent le mouvement dans le sens antéro-postérieur, ils privilégient alors une observation du mouvement dans le plan sagittal. Ils font ainsi émerger des structures signifiantes qui leur permettent d'intervenir auprès des gymnastes. Par ailleurs, les entraîneurs sont parfois en phase d'enquête afin de remédier à des «pannes » d'interprétation de certaines réalisations. Leurs stratégies d'enquête visent, par une recherche en amont, à établir des relations causales diachroniques, afin de déterminer une séquence sur laquelle intervenir. Puis, la recherche se porte au sein de la phase de placement retenue afin d'intervenir sur ses propriétés. Percevoir serait ainsi faire émerger des relations logiques entre certains aspects du mouvement.

\section{2. 2. Des gestalts dynamiques}

Pour les gestaltistes, l'organisation perceptive consiste à établir dans la mosaïque visuelle une discrimination entre des éléments auxquels on accorde le statut de forme ou de figure et d'autres qui constituent le fond ou l'environnement (Koffka, 1935). L'analyse de l'activité des entraîneurs en situation montre qu'ils ne perçoivent pas des formes statiques mais des totalités dynamiques qui intègrent certes des formes mais aussi des forces, des trajectoires, des vitesses, des déplacements. Ces gestalts, élaborées au cours de l'activité de transmission, permettent alors à l'entraîneur, à partir d'éléments discrets perçus, de caractériser une phase de placement ou de prédire l'évolution d'une habileté gymnique. Ainsi, le placement de la tête au cours d'une sortie à la barre fixe est typiquement associé pour l'entraîneur $E$ à un lâcher de barre trop hâtif :

" tu lâches trop tôt parce que tu tires la tête et donc tu ne peux pas t'enrouler longtemps avant de lâcher. La tête en extension, ça te fait lâcher ”.

Cette identification est une synthèse par détermination réciproque du tout et des parties, la perception étant «d'emblée perception de relations car une relation n'est pas nécessairement le fruit d'une opération intellectuelle, ni même postérieure à la perception des termes qu'elle relie. » (Rosenthal \& Visetti, 1999).

\section{Discussion}

Pour les gestaltistes, l'émergence d'une forme se produit par un processus de «groupement spontané » des stimuli du champ sensoriel qui sont perçus comme des ensembles structurés, à partir de certains principes naturels d'organisation, tels que les principes de proximité ou de similitude. Le caractère expérientiel des gestalts qui émergent au cours de l'activité de transformation des habiletés gymniques remet en question un certain nombre de postulats de l'organisation perceptive définis par les 
gestaltistes. Ces postulats initiaux stipulent entre autres que la gestalt ne résulte pas d'inférences perceptives, qu'elle n'est pas tributaire de l'expérience. Cependant, certains auteurs (Pomerantz \& Kubovy, 1981) ont avancé l'idée d'une loi de vraisemblance qui associerait l'émergence de formes à leur probabilité d'apparition dans l'environnement. Ce principe de vraisemblance constituerait un principe d'économie d'information qui intègrerait la dimension expérientielle de l'activité perceptive. Ce fonctionnement économique est représentatif du fonctionnement efficace des experts. En s'intéressant au enseignants experts, Faingold (1996) a notamment relevé que leur efficacité reposait en partie sur un fonctionnement métonymique a minima, élégant en ce qu'il est caractérisé par une grande économie de moyens. Les connaissances des entraîneurs experts pourraient aussi reposer sur des archétypes métonymiques, constitués de saillances perceptives permettant de rapporter la totalité dynamique du mouvement à l'une de ses phases ou à l'un des aspects discrets la composant.

Une compréhension et une explicitation plus précises de ces archétypes restent à effectuer. Elles pourraient alimenter des dispositifs de formation des entraîneurs novices par des entraîneurs expérimentés dans le cadre du mentoring. En effet, ces archétypes sont au fondement de la connaissance mécanique empirique du mouvement des entraîneurs. Ces derniers les mobilisent pour se rendre intelligibles les mouvements corporels complexes sur lesquels ils ont à travailler. Néanmoins le caractère fortement implicite de ces connaissances les rend difficilement transmissibles et il faut de nombreuses années, des centaines d'heures de présence dans les salles de gymnastique pour les acquérir. Leur identification et leur formalisation devrait permettre, d'une part, de les rendre plus facilement transmissibles, et, d'autre part, de les constituer comme corpus de savoirs de métier. Sur ce dernier point en effet, le passage d'un savoir tacite dans une communauté professionnelle à un corpus formalisé, devrait favoriser sa diffusion, mais également la possibilité d'un travail de révision critique, de débat, de controverses, c'est-àdire une dynamique sociale centrée sur les savoirs professionnels. 


\section{Bibliographie}

Cizeron, M. (2002). Croyances factuelles et croyances représentationnelles : les bases anthropo-cognitives de l'expertise en enseignement scolaire de la gymnastique. Thèse non publié de doctorat en STAPS, Université Rennes 2.

Faingold, N. (1996). Du stagiaire à l'expert : construire les compétences professionnelles. In P. Perrenoud, P. (dir.) Former des enseignants professionnels. Louvain La Neuve : de Boeck.

Fédération Française de Gymnastique. (1997). Mémento 1997 des activités gymniques. Toulouse : Cota Production.

Greeno, J. G. (1998). The situativity of knowing, learning and research. American Psychologist, 53, 1, 5-26.

Kirshner, D., \& Whitson, J.A. (1997). Situated cognition: social, semiotic, and psychological perspectives, Mahwah, NJ, Erlbaum.

Koffka, K. (1935). Principles of Gestalt Psychology. New-York : Harcourt, Brace.

Le Boterf, G. (1998). L’ingénierie des compétences. Editions d'organisation.

Malglaive, G. (1990). Enseigner à des adultes. Paris : PUF.

Oddone, I., Re, A., \& Briante, G. (trad.Fr, 1981). Redécouvrir l'expérience ouvrière. Vers une autre psychologie du travail ? Paris : Editions Logiques.

Passeron, J.C., \& Revel, J. (dir.) (2005). Penser par cas. Paris : Éditions de l'EHESS.

Pernet, M. (1994). Gymnastique artistique et sportive. " 9 techniques de base à la loupe ». Dossier EPS, 18. Editions Revue EPS.

Piard, C. (1992).Science et technique de l'éducation gymnique. Paris : PUF.

Pomerantz, J.R., \& Kubovy, M. (1981). Perceptual organization : An overview. In M. Kubovy (dir). Perceptual organization, 423-456, Hillsdale, NJ : Lawrence Erlbaum.

Rolland, C. (2004). Les «phases de placement » comme formes efficaces reconnues par les entraîneurs experts en gymnastique. Mémoire de DEA. Université de Technologie de Compiègne.

Rolland,C., \& Cizeron,M. (2008). Le modelage du corps en mouvement des gymnastes par les entraîneurs experts en gymnastique artistique. Revue Interrogations 7.

Rosenthal, V., \& Visetti, Y.M., (1999). Sens et temps de la gestalt. Intellectica, 1, 147-227.

Saury, J., Ria, L., Sève, C., \& Gal-Petitfaux, N. (2006). Action ou cognition située : enjeux scientifiques et intérêts pour l'enseignement de l'EPS. Revue EP.S., 321, 5-11.

Schön, D.A. (1983). The reflective practionner. New-York. Basic Books. 
Smith, T. (1991). Biomécanique et gymnastique. Paris : PUF.

Strauss, A. L. (1992). La méthode comparative continue en analyse qualitative. In I. Baszanger (Ed.), La trame de la négociation, sociologie qualitative et interactionnisme (pp. 283-311). Paris : L'Harmattan.

Suchman, L. (1987). Plans and Situated Actions: the problem of human-machine interaction. Cambridge: Cambridge University Press.

Theureau, J. (1992). Le cours d'action : analyse sémio-logique. Essai d'une anthropologie cognitive située. Berne : Peter-Lang.

Vermersch, P. (1994). L'entretien d'explicitation. Paris : ESF. 